\title{
Syphilis in the Americas: a protocol for a systematic review of syphilis prevalence and incidence in four high-risk groups, 1980-2016
}

Ken Kitayama ${ }^{1 *}$ (D), Eddy R. Segura ${ }^{2,3}$, Jordan E. Lake ${ }^{4}$, Amaya G. Perez-Brumer ${ }^{5}$, Catherine E. Oldenburg ${ }^{6}$, Bethany A. Myers ${ }^{7}$, Paria Pourjavaheri ${ }^{2}$, Chinomnso N. Okorie ${ }^{8}$, Robinson L. Cabello ${ }^{9}$ and Jesse L. Clark ${ }^{2}$

\begin{abstract}
Background: Syphilis infection has recently resurfaced as a significant public health problem. Although there has been a tremendous amount of research on the epidemiology of syphilis, there has been limited work done to synthesize the extensive body of research and systematically estimate patterns of disease within high-risk groups in the Americas. The purpose of this systematic review and meta-analysis is to (1) summarize recent patterns of syphilis infection in North and South America among four high-risk groups (MSM, transgender women, sex workers, and incarcerated individuals) from 1980 to 2016, (2) identify and differentiate regional geographic epidemiologic characteristics, and (3) compare the epidemics of the economically developed countries of North America from the developing countries and public health systems of Latin America and the Caribbean.

Methods/design: Primary studies reporting syphilis prevalence and/or incidence in at least one of the four high-risk groups will be identified from Medline/PubMed, Embase, Lilacs, SciELO, The Cochrane Library, Web of Science, Scopus, ProQuest, CINAHL, Clase, and Periódica, as well as "gray" literature sources (conference abstracts, country reports, etc.). Studies published from 1980 through 2016 will be included. Data will be extracted from studies meeting inclusion and exclusion criteria and a random effects meta-analysis of prevalence and incidence estimates will be conducted. Heterogeneity, risk of bias, and publication bias will be assessed. Pooled prevalence and incidence estimates will be calculated for comparisons based on geographic region, risk factors, and time period.
\end{abstract}

Discussion: Our systematic review and meta-analysis aims to contribute to an improved understanding of global epidemiologic patterns of syphilis infection in most-at-risk populations. Through systematic classification of the existing literature, and comparison of disease patterns across regional, temporal and socio-behavioral differences, we hope to improve public health surveillance and improve efforts to control the spread of disease across the Americas.

Systematic review registration: PROSPERO CRD42016047306.

Keywords: Syphilis, Prevalence, Incidence, Men who have sex with men, Transgender women, Sex workers, Incarcerated individuals, Systematic review, Meta-analysis, Protocol

\footnotetext{
*Correspondence: KKitayama@mednet.ucla.edu

1 Department of Medicine, Division of Infectious Diseases, UCLA South

American Program in HIV Prevention Research (SAPHIR), David Geffen School

of Medicine at UCLA, 10833 Le Conte Ave, CHS 37-121, Los Angeles, CA

90095, USA

Full list of author information is available at the end of the article
} 


\section{Background}

\section{Global burden of syphilis}

Syphilis is a critical public health problem worldwide. Although the incidence of syphilis infection in the USA and Western Europe decreased after the introduction of penicillin in the early 1940s [1, 2], a resurgence in syphilis transmission that began in the 1980s has now gained substantial strength. Beginning in the 2000s, a growing number of isolated syphilis outbreaks were reported in North America, Europe, and Australia, primarily within sexual networks of men who have sex with men (MSM), and often associated with HIV co-infection or cotransmission [3-11]. Following the successful introduction of biomedical approaches to HIV prevention, these sporadic outbreaks have more recently grown in frequency and size to mark a new era of syphilis transmission. In contrast to the relatively more effective control of disease in the USA and Western Europe, endemic rates of syphilis transmission have been stubbornly persistent in the resource-limited public health systems of Latin America, Asia, and Africa [12-21]. Despite the proliferation of epidemiologic reports on syphilis prevalence and incidence, we are not aware of any comprehensive synthesis of the available literature that can (i) summarize recent patterns of syphilis infection and transmission, (ii) identify and differentiate regional epidemiologic characteristics, and (iii) compare the developing syphilis epidemics of the economically developed countries of North America with the endemic patterns of disease observed in Latin America and the Caribbean.

The global epidemiology of syphilis infection varies significantly by geography. A 2015 systematic review based on data from the 2012 Global AIDS Response Progress Reporting (GARPR) system estimated there were approximately $17,721,000$ cases of syphilis among men and women 15-49 years of age worldwide [22]. Though country-specific estimates were not provided, the study authors noted differences in syphilis prevalence according to World Bank economic classifications, with prevalence estimates lowest for high-income countries, at $0.2 \%$ overall, and ranging from $0.3 \%$ to $1.3 \%$ in middle- to lowincome economies.

\section{Syphilis in high-risk groups}

Risks for syphilis infection among MSM, transgender women (TW), and sex workers (SW) of all genders are disproportionately high across geographic locations, but there have been few comprehensive global estimates of disease prevalence and incidence in these key populations [23-25]. GARPR did not begin collecting behavioral data on syphilis in adults until 2012, with a formal roll out of reporting for all regions initiated in 2013 [26]. As a result, pre-2012 GARPR estimates of syphilis infection among MSM and TW were based on extrapolations from reported data on cases of maternal and congenital syphilis, with the assumption that heterosexual transmission would result in a 1:1 male to female ratio of syphilis prevalence [22]. In Latin America and the Caribbean, a 2013 systematic review of syphilis data in MSM, TW, SW, and clients of SW included 35 studies on syphilis prevalence in MSM, with a prevalence $>4 \%$ in three quarters of the estimates included and $>7.5 \%$ in half [25]. Five of those studies included TW, with syphilis prevalence ranging from $6.5 \%$ in El Salvador to $43.3 \%$ in Brazil. The same review identified 49 studies with female SWs, half of them with a syphilis prevalence $>5 \%$, but noted large variations between the populations sampled.

In addition to MSM and SW, the United Nations has also identified incarcerated individuals as a group at high-risk for syphilis and other sexually transmitted infections (STIs), with frequencies of disease two to 10 times higher (and potentially 50 times greater) among prisoners than in the general population worldwide [27]. A 2012 systematic review of incarcerated populations calculated pooled syphilis prevalence estimates of $2.5 \%$ in men and $6.1 \%$ in women, finding that the incidence of infection among women was 2.7 times greater than in men (95\% CI 1.5-4.8) [28].

\section{Review aims}

In order to improve the public health response to the emerging syphilis epidemics in the Americas, detailed knowledge of epidemiologic patterns of disease in mostat-risk populations is needed. A systematic review and analysis of the broad range of existing data will help to establish a framework for understanding the parameters of syphilis transmission across the region and help to define target points for STI control efforts. Comparing patterns of disease in Latin America and the Caribbean with those seen in Canada and the USA will help to illuminate how behavioral, biological, social, and structural characteristics of syphilis transmission differ across geographic, socioeconomic, and epidemiologic contexts, as well as how they may have changed over time. Ultimately, the improved understanding of the global epidemiology of syphilis infection to be provided by our analysis will help to define and allocate future public health surveillance and control efforts. We plan to conduct a systematic review and meta-analysis of data on syphilis infection prevalence and incidence in four high-risk groups (MSM, TW, SW, and incarcerated persons) within Latin America, the Caribbean, and North America (USA and Canada), in published studies and reports from 1980 to 2016.

\section{Methods and design}

This systematic review and meta-analysis protocol was developed according to the Preferred Reporting Items 
for Systematic Reviews and Meta-Analysis Protocols (PRISMA-P) 2015 statement [29]. The protocol has been published in the PROSPERO International Prospective Register of systematic reviews (http://www.crd.york.ac.uk/ PROSPERO), registration number CRD42016047306.

\section{Criteria for considering studies for review Inclusion criteria}

1. Studies reporting primary, quantitative data on the prevalence and/or incidence (cumulative incidence or incidence rates) of active and/or lifetime syphilis infection among at least one high-risk group (MSM, TW, SW, and/or incarcerated persons) who reside in countries and territories within the Americas and who are from any race, ethnicity, socioeconomic status, and/or level of education will be included. For the purpose of this study, "The Americas" refers to all countries designated under this geographical group by the World Health Organization [30].

2. Included studies must describe appropriate criteria used for diagnosis of active syphilis infection, including serologic assays (e.g., rapid plasma reagin (RPR), venereal disease research laboratory test (VDRL), treponema pallidum particle agglutination assay (TPPA), microhemagglutination assay (MHA-TP), treponema pallidum enzyme-linked immunosorbent assay (TP-EIA)), dark-field microscopy, and/or clinical diagnosis based on history and physical exam findings.

3. Articles published in English, Spanish, Portuguese, or French or with sufficient information for data abstraction from an English abstract will be considered for inclusion.

We plan to collect data from articles and reports published from 1 January 1980 through 31 December 2016. This date range selection is based on the fact that in the USA, the highest recorded incidence of syphilis since 1950 was observed in the year 1982 [31], with rates initially declining, but peaking again in 1990-1991, thought to be because of a new surveillance case definition for congenital syphilis [32]. Syphilis infection rates then declined thereafter in association with increased public health efforts to prevent HIV/AIDS and reduce AIDSrelated mortality $[33,34]$ and subsequently followed by a resurgence of syphilis infection among MSM beginning in the late 1990s and early 2000s [35-37].

\section{Exclusion criteria}

1. Studies or reports that include mixed prevalence or incidence data on syphilis infection as a composite outcome with another STI and that do not specifically report data on syphilis infection alone.
2. Studies or reports that extrapolate syphilis prevalence or incidence data in high-risk groups from general population statistics or congenital syphilis data without direct measurement of the social or behavioral factors used for classification.

3. Publications that report the same data in multiple sources. If the same data is published in more than one article, report, or abstract, the publication reporting the most complete version of the data will be used.

4. Narrative reviews, opinion pieces, letters, or other publications that do not report primary data and/or do not provide sufficient description of the study methodology. Case series and case reports, as well as mathematical modeling studies based on previously reported data, will be excluded.

\section{Search strategy for identification of relevant studies}

The search strategy will be composed of two stages:

Stage 1: bibliographic database review

Ten electronic databases (including MEDLINE/PubMed, Embase, Lilacs, SciELO, The Cochrane Library, Web of Science, Scopus, ProQuest, CINAHL, Clase, and Periódica) will be searched for articles published from January 1, 1980, through December 31, 2016. A list of search terms including "syphilis" or "Treponema pallidum" or "T. pallidum" or "pallidum" in addition to terms for each of the four highrisk groups of interest (MSM, TW, SW, and incarcerated persons) was generated based on strategies from previously published systematic reviews [28, 38-41]. When possible, controlled search terms $(\mathrm{MeSH})$ will be combined with text words. Otherwise, text words alone will be searched. Reference lists of all included articles will be hand-searched to identify additional studies and reports to include in our analysis. For the full search strategy, see Additional file 1.

Stage 2: gray literature search

The Google Scholar search engine will be used to identify "gray" literature (documents not published by commercial publishers such as agency reports, government articles, and academic theses) as previously described [42]. As the Google Scholar search string is limited to 256 characters, a separate search will be conducted for each of the four high-risk groups, and the first 100 results for each of the searches will be reviewed. Conference abstract archives or abstract books will be searched for the following conferences: International AIDS Conference, IAS Conference on HIV Pathogenesis, Treatment and Prevention, Conference on Retroviruses and Opportunistic Infections, IDWeek, and the International Society for Sexually Transmitted Diseases Research. GARPR system country reports, Centers for Disease Control and the Public Health Agency of Canada statistics reports will also be searched. Additionally, we will also poll recognized experts in the field for additional citations or references not identified using other methods. 
Selecting studies for inclusion Studies that are found utilizing the search strategies described above will be compiled with their titles and abstracts into EndNote X7 (Thomson Reuters). As EndNote's ability to accurately remove duplicates has been found to be sub-optimal [43, 44], de-duplication will be performed using Mendeley (Elsevier), which has been found to report fewer false negatives and similar false positives in its de-duplication results when compared to EndNote [45].

The primary author (KK) will apply the inclusion and exclusion criteria to the de-duplicated list of articles, and a separate reviewer $(\mathrm{CO})$ will conduct an independent screen of a random selection of $10 \%$ of all titles. Comparison of the two authors' screen results will be used to calculate an inter-rater reliability score using Cohen's kappa coefficient [46]. The initial screens will result in the creation of three categories: (1) Included, (2) Excluded, or (3) Pending (when the article initially appears to incompletely fit exclusion and/or inclusion criteria). Titles classified as "Pending" will be examined by a separate author with specialized expertise in the clinical and epidemiologic classification of syphilis infection (JLC) who will make the final determination of inclusion or exclusion. In the full text screen, two reviewers (KK and PP) will independently review the full article texts and apply the inclusion and exclusion criteria listed above. Any disagreements in categorizations within the full-text screen will be resolved by group adjudication. A PRISMA flow diagram outlining the procedures described above will be prepared for transparency and to provide a visual overview of how the final list of articles for data collection was constructed.

Data collection Two reviewers (KK and PP) will independently extract and manage the data for the final set of included studies. A customizable electronic data extraction form will be created using form.io (Dallas, TX) that will be piloted and edited through an iterative process using feedback from the entire panel of authors. Any disagreements in data extraction between KK and PP will be discussed and resolved, with the senior author (JLC) acting as arbiter in instances where disagreements cannot be resolved.

Data items The following data will be extracted:

1. Publication details: year, title, first author, language, type of publication.

2. Study design: study type (cross-sectional, cohort, clinical trial, etc.), sampling method (probability or non-probability sampling and specific type of sampling), recruitment site type (for non-probability sampling).
3. Study participants: city and country where study took place, study time frame, participants' sex and/ or gender, mean/median age and age range, primary (e.g., MSM, TW, SW, incarcerated) and secondary (e.g., mobile app/Internet use to find sex partners, IV drug use) risk-group characteristic(s), number of persons surveyed or studied for per group, HIV serostatus (when available), race, and ethnicity. Diagnostic criteria used to define syphilis infection, syphilis re-infection, and neurosyphilis (if applicable). Number and frequency of syphilis cases, including stage at diagnosis, number and frequency of cases of syphilis re-infection, and number and frequency of cases of neurosyphilis. Reported titers of diagnostic tests (e.g., RPR, VDRL) will be recorded when available.

4. Outcome measures: reported estimates (or, if sufficient raw data is available, estimates calculated by our study team) of the point prevalence, period prevalence, cumulative incidence, and/or incidence rate of active and/or lifetime syphilis in selected areas. Lifetime syphilis will be defined within extracted data as (1) any positive non-treponemal test with confirmatory treponemal test or (2) selfreported lifetime history of syphilis infection. Active syphilis will be defined as (1) a four-fold increase in non-treponemal test compared to previous results with positive confirmatory treponemal test or (2) a positive non-treponemal test (e.g., RPR or VDRL) titer of $1: 8$ or greater with a positive confirmatory treponema-specific test (e.g., TPPA or MHA-TP).

\section{Critical appraisal of included studies}

Quality assessment of the studies will be performed using a nine-item critical appraisal tool to assess the validity and rate the methodological quality of studies reporting prevalence data [47]. Compared to other instruments specifically designed to assess a narrower scope of study designs, this tool is designed to qualify studies of varied designs reporting prevalence data [48]. KK and PP will each independently apply this quality assessment tool to all included studies. Any disagreements will be resolved by consensus discussion and in consultation with the senior author (JLC). The final assessment of risk of bias for all included publications will be presented in a table. Publication bias will be assessed using funnel plots of the study outcome against the sample size, as described by Egger et al. [49]. Furthermore, GRADE guidelines for assessing publication bias will be referred to for additional guidance [50].

\section{Data synthesis}

We will prepare a narrative synthesis and quantitative meta-analysis of the prevalence and incidence of active and lifetime syphilis in the four previously defined highrisk groups in the Americas, with all relevant data 
presented in tables. Estimates from studies found to be of sufficient quality will be pooled to estimate overall prevalence and incidence of active syphilis infection and overall prevalence and incidence of any syphilis infection, presenting meta-analysis results with forest plots. Data will be displayed graphically as needed to assist in the interpretation of data not amenable to the application of statistical tests for trends.

Because we anticipate elevated levels of heterogeneity in our included studies in terms of study design, outcome measurement, and follow-up (for incidence rates), we will conduct meta-analyses using a DerSimonian and Laird random effects model and will account for heterogeneity of studies by including a parameter for inter-study variation. We will combine the percentages of patients with lifetime and/or active syphilis (as defined above) in each individual study to calculate pooled prevalence estimates along with $95 \%$ confidence intervals (CI), using a double arcsine transformation of prevalence.

We will conduct separate subgroup analyses of the data extracted from all studies: (1) between-group analyses of the four high-risk groups (MSM, TW, SW, prisoners) by calculating estimates separately for each group and investigating differences, (2) differences between geographic sub-regions (Latin America and the Caribbean versus North America), (3) changes in syphilis prevalence and incidence over time, as stratified by 10 -year intervals, (4) estimates within specific subgroups according to HIV serostatus; and (5) differences in estimates depending on a diagnostic method (self-reported history, serologic testing strategy). Heterogeneity will be assessed with $I^{2}$ and $\tau^{2}$ statistics. Sources of heterogeneity between included studies will be examined by stratifying data according to study characteristics. All analyses will be conducted using Stata 14.0 (StataCorp, College Station, TX).

\section{Discussion}

This systematic review and meta-analysis of syphilis in the Americas will compile and analyze the available epidemiologic literature, summarizing the burden of syphilis in high-risk groups in Latin America, the Caribbean, the USA, and Canada. We will collect prevalence and incidence data on active and lifetime syphilis in four high-risk groups (MSM, TW, SW, incarcerated persons), comparing and contrasting geographic regions within the Americas and across time periods from 1980 to 2016 . We will also aim to better characterize details and potential nuances of the current epidemiology of syphilis transmission, including the impact on disease incidence of new biomedical prevention technologies like pre-exposure prophylaxis, the use of Internet/mobile applications to find sex partners, and rates of HIV co-infection.

Based on an informal, preliminary review of the existing literature, we expect that there will be tremendous diversity in the prevalence and incidence of syphilis infection between high-risk groups in the USA and Canada as compared with Latin America and the Caribbean. To guide the analysis, we propose the following hypotheses: (1) The prevalence and incidence of syphilis infection in Latin America and the Caribbean will be significantly higher than the prevalence/incidence of infection in the USA and Canada; (2) The majority of syphilis cases reported in the USA and Canada will be identified during primary or secondary syphilis infection (diagnosed through symptomatic illness or network tracing), while the majority of cases in Latin America and the Caribbean will be diagnosed as latent disease (asymptomatic cases identified through serologic testing); and (3) In all regions, there will be a close epidemiologic association between syphilis infection and HIV acquisition and transmission.

Our systematic review and meta-analysis aims to contribute to an improved understanding of global epidemiologic patterns of syphilis infection in most-at-risk populations. Through systematic classification of the existing literature and comparison of disease patterns across regional, temporal, and socio-behavioral differences, we hope to improve public health surveillance and improve efforts to control the spread of disease across the Americas. Understanding the prevalence and incidence of syphilis within and between these high-risk groups will help guide resource allocation and educational efforts, particularly in resource-limited settings in Latin America and the Caribbean.

\section{Additional file}

Additional file 1: Search strategy. (DOCX $106 \mathrm{~kb})$

\section{Abbreviations}

Cl: Confidence interval; GARPR: Global AIDS Response Progress Report; MHA-TP: Microhemagglutination assay; MSM: Men who have sex with men; RPR: Rapid plasma reagin; STI: Sexually transmitted infections; SW: Sex workers; TP-EIA: Treponema pallidum enzyme-linked immunosorbent assay; TPPA: Treponema pallidum particle agglutination assay; TW: Transgender women; VDRL: Venereal disease research laboratory test

\section{Acknowledgements}

During the elaboration of this project, KK was a research fellow in the $\mathrm{NIH}$ funded South American Program in HIV Prevention Research (SAPHIR) of the David Geffen School of Medicine at UCLA and gratefully acknowledges the support he received from the staff in both Lima and Los Angeles.

\section{Availability of data and materials}

The datasets analyzed during the current study will be made available from the corresponding author on reasonable request.

\section{Authors' contributions}

JLC conceived of the study, drafted the manuscript, and provided critical review of the manuscript. KK furnished the details of the study design, developed the search strategy, conducted the pilot search, drafted the manuscript, and provided the critical review of the manuscript. ERS and CEO provided the statistical advice, participated in the data synthesis design, and edited the manuscript. JEL contributed to the details of the study design, drafted the manuscript, and provided the critical review of the manuscript. AGPB contributed to the details of the study design and 
edited the manuscript. BAM participated in the development of the search strategy, assisted with the pilot search, and contributed to refining the details of the study design. PP and CNO participated in the pilot search and contributed to refining the details of the study design. RLC provided specialist expertise and edited the manuscript. All authors read and approved the final manuscript.

\section{Funding}

This research was supported by the National Institutes of Health grants R25 MHO87222 to JLC and K23Al110532 to JEL. The funders had no role in protocol design, decision to publish, or preparation of the manuscript.

\section{Ethics approval and consent to participate}

Not applicable

\section{Consent for publication}

Not applicable

\section{Competing interests}

The authors declare that they have no competing interests.

\section{Publisher's Note}

Springer Nature remains neutral with regard to jurisdictional claims in published maps and institutional affiliations.

\section{Author details \\ 'Department of Medicine, Division of Infectious Diseases, UCLA South American Program in HIV Prevention Research (SAPHIR), David Geffen School of Medicine at UCLA, 10833 Le Conte Ave, CHS 37-121, Los Angeles, CA 90095, USA. ${ }^{2}$ Department of Medicine, Division of Infectious Diseases, David Geffen School of Medicine at UCLA, Los Angeles, CA, USA. ${ }^{3}$ Escuela de Medicina, Universidad Peruana de Ciencias Aplicadas, Lima, Peru. ${ }^{4}$ Department of Medicine, Division of Infectious Diseases, McGovern Medical School at the University of Texas Health Science Center at Houston, Houston, TX, USA. ${ }^{5}$ Columbia University Mailman School of Public Health, New York, NY, USA. 'San Francisco School of Medicine, Francis I. Proctor Foundation for Research in Ophthalmology, Department of Ophthalmology, and Department of Epidemiology and Biostatistics, University of California, San Francisco, CA, USA. 'Louise M. Darling Biomedical Library at UCLA, Los Angeles, CA, USA. ${ }^{8}$ Department of Biology, San Francisco State University, San Francisco, CA, USA. ${ }^{9}$ Asociación Civil Vía Libre, Lima, Peru.}

\section{Received: 26 April 2017 Accepted: 2 October 2017}

\section{Published online: 10 October 2017}

\section{References}

1. Mahoney JF, Arnold RC, Sterner BL, Harris A, Zwally MR. Landmark article Sept 9, 1944: penicillin treatment of early syphilis: II. By J.F. Mahoney, R.C. Arnold, B.L. Sterner, A. Harris and M.R. Zwally. JAMA. 1984;251(15):2005-10.

2. Holmes KK. Azithromycin versus penicillin $\mathrm{G}$ benzathine for early syphilis. $\mathrm{N}$ Engl J Med. 2005;353(12):1291-3.

3. Bernstein KT, Tulloch R, Montes J, Bolan G, Dyer IE, Lawrence M, Kaur AP, Kodagoda $\mathrm{D}$, Rotblatt $\mathrm{H}$, Kerndt $\mathrm{P}$, et al. Outbreak of syphilis among men who have sex with men-Southern California, 2000 (Reprinted from MMWR, vol 50, pg 117-120, 2001). Jama-J Am Med Assoc. 2001;285(10):1285-7.

4. Cowan S. Syphilis in Denmark-outbreak among MSM in Copenhagen, 2003-2004. Euro Surveill. 2004;9(12):25-7.

5. D'Souza G, Lee JH, Paffel JM. Outbreak of syphilis among men who have sex with men in Houston, Texas. Sex Transm Dis. 2003;30(12):872-3.

6. de Luise C, Blank S, Brown J, Rubin S, Meyers A, Neylans L, Paz-Bailey G, Markowitz L, Cdc. Primary and secondary syphilis among men who have sex with men-New York City, 2001 (Reprinted from MMWR, vol 51, pgs 853-856, 2002). Jama-J Am Med Assoc. 2002;288(15):1840-2.

7. Emerson CR, Lynch A, Fox R, Smyth B, Gray S, Dinsmore WW, Maw RD. The syphilis outbreak in Northern Ireland. Int J STD AIDS. 2007;18(6):413-7.

8. Giuliani M, Palamara G, Latini A, Maini A, Di Carlo A. Evidence of an outbreak of syphilis among men who have sex with men in Rome. Arch Dermatol. 2005;141(1):100-1.

9. Jayaraman GC, Read RR, Singh A. Characteristics of individuals with male-tomale and heterosexually acquired infectious syphilis during an outbreak in Calgary, Alberta, Canada. Sex Transm Dis. 2003;30(4):315-9.
10. Poulton M, Dean GL, Williams DI, Carter P, Iversen A, Fisher M. Surfing with spirochaetes: an ongoing syphilis outbreak in Brighton. Sex Transm Infect. 2001;77(5):319-21.

11. Ruiz-Sancho A, Barreiro P, Castellares C, Labarga P, Ramos B, GarciaSamaniego J, Gutierrez M, Soriano V. Outbreak of syphilis, but not of acute hepatitis C, among HIV-infected homosexual men in Madrid. HIV Clin Trials. 2007:8(2):98-101.

12. Ahmed HJ, Omar K, Adan SY, Guled AM, Grillner L, Bygdeman S. Syphilis and human immunodeficiency virus seroconversion during a 6-month follow-up of female prostitutes in Mogadishu, Somalia. Int I STD AIDS. 1991;2(2):119-23.

13. Killewo JZ, Sandstrom A, Bredberg Raden U, Mhalu FS, Biberfeld G, Wall S. Prevalence and incidence of syphilis and its association with HIV-1 infection in a population-based study in the Kagera region of Tanzania. Int J STD AIDS. 1994;5(6):424-31.

14. Kumar B, Rajagopalan M, Sehgal S, Sharma M, Malhotra S. Syphilis serology and human immunodeficiency virus positivity in Chandigarh. Int J STD AIDS. 1990; 1(6):438-9.

15. Kunawararak P, Beyrer C, Natpratan C, Feng W, Celentano DD, de Boer M, Nelson KE, Khamboonruang C, Deboer M, Nelson KE, et al. The epidemiology of HIV and syphilis among male commercial sex workers in northern Thailand. AIDS. 1995;9(5):517-21.

16. Limpakarnjanarat K, Mastro TD, Saisorn S, Uthaivoravit W, Kaewkungwal J, Korattana S, Young NL, Morse SA, Schmid DS, Weniger BG, et al. HIV-1 and other sexually transmitted infections in a cohort of female sex workers in Chiang Rai, Thailand. Sex Transm Infect. 1999;75(1):30-5.

17. Nelson KE, Eiumtrakul S, Celentano D, Maclean I, Ronald A, Suprasert S, Hoover DR, Kuntolbutra S, Zenilman JM. The association of herpes simplex virus type 2 (HSV-2), Haemophilus ducreyi, and syphilis with HIV infection in young men in northern Thailand. J Acquir Immune Defic Syndr Hum Retrovirol. 1997;16(4):293-300.

18. Lurie P, Fernandes ME, Hughes V, Arevalo El, Hudes ES, Reingold A, Hearst N. Socioeconomic status and risk of HIV-1, syphilis and hepatitis B infection among sex workers in Sao Paulo State, Brazil. Instituto Adolfo Lutz Study Group. AIDS. 1995;9(Suppl 1):S31-7.

19. Trujillo L, Muñoz D, Gotuzzo E, Yi A, Watts DM, Munoz D, Gotuzzo E, Yi A, Watts DM. Sexual practices and prevalence of HIV, HTLV-I/II, and Treponema pallidum among clandestine female sex workers in Lima, Peru. Sex Transm Dis. 1999;26(2):115-8.

20. Uribe-Salas F, Del Río-Chiriboga C, Conde-Glez CJ, Juárez-Figueroa L, UribeZúñaga P, Calderón-Jaimes E, Hernández-Avila M, UribeSalas F, DelRioChiriboga C, CondeGlez CJ, et al. Prevalence, incidence, and determinants of syphilis in female commercial sex workers in Mexico City. Sex Transm Dis. 1996;23(2):120-6.

21. Venegas VS, Madrid JP, Lorenzana I, Grillner L, Cosenza H, Bygdeman S. Human immunodeficiency virus infection and syphilis in Hondurian female prostitutes. Int J STD AIDS. 1991;2(2):110-3.

22. Newman L, Rowley J, Vander Hoorn S, Wijesooriya NS, Unemo M, Low N, Stevens G, Gottlieb S, Kiarie J, Temmerman M. Global estimates of the prevalence and incidence of four curable sexually transmitted infections in 2012 based on systematic review and global reporting. PLoS One. 2015; 10(12):e0143304

23. Guidance on syphilis testing in Latin America and the Caribbean: improving uptake, interpretation, and quality of testing in different clinical settings. Washington, DC: PAHO; 2015.

24. Force USPST, Bibbins-Domingo K, Grossman DC, Curry SJ, Davidson KW, Epling JW Jr, Garcia FA, Gillman MW, Harper DM, Kemper AR, et al. Screening for syphilis infection in nonpregnant adults and adolescents: US preventive services task force recommendation statement. JAMA. 2016;315(21):2321-7.

25. Zoni AC, Gonzalez MA, Sjogren HW. Syphilis in the most at-risk populations in Latin America and the Caribbean: a systematic review. International journal of infectious diseases : IJID : official publication of the International Society for Infectious Diseases. 2013;17(2):e84-92.

26. Report on global sexually transmitted infection surveillance 2013. In. Geneva: WHO; 2013.

27. HIV prevention, treatment and care in prisons and other closed settings: a comprehensive package of interventions. Vienna: United Nations Office on Drugs and Crime; 2012.

28. Kouyoumdjian $F G$, Leto $D$, John $S$, Henein $H$, Bondy S. A systematic review and meta-analysis of the prevalence of chlamydia, gonorrhoea and syphilis in incarcerated persons. Int J STD AIDS. 2012;23(4):248-54. 
29. Moher D, Shamseer L, Clarke M, Ghersi D, Liberati A, Petticrew M, Shekelle P Stewart LA, Group P-P. Preferred reporting items for systematic review and meta-analysis protocols (PRISMA-P) 2015 statement. Syst Rev. 2015;4:1.

30. World Health Statistics 2016. Monitoring Health for the SDGs, Sustainable Development Goals. Geneva: World Health Organization; 2016.

31. Centers for Disease C. Syphilis and congenital syphilis_United States, 1985-1988. MMWR Morb Mortal Wkly Rep. 1988;37(32):486-9.

32. Division of STD Prevention. Sexually transmitted disease surveillance 2000 syphilis surveillance report. Atlanta: Department of Health and Human Services, Centers for Disease Control and Prevention (CDC); 2000.

33. Centers For Disease C. Syphilis—United States, 1983. MMWR Morb Mortal Wkly Rep. 1984;33(30):433-6. 441

34. Chesson HW, Dee TS, Aral SO. AIDS mortality may have contributed to the decline in syphilis rates in the United States in the 1990s. Sex Transm Dis. 2003:30(5):419-24.

35. Centers for Disease C, Prevention. Resurgent bacterial sexually transmitted disease among men who have sex with men-King County, Washington, 1997-1999. MMWR Morb Mortal Wkly Rep. 1999;48(35):773-7.

36. Centers for Disease C, Prevention. Outbreak of syphilis among men who have sex with men-Southern California, 2000. MMWR Morb Mortal Wkly Rep. 2001;50(7):117-20.

37. Centers for Disease C, Prevention. Primary and secondary syphilis among men who have sex with men-New York City, 2001. MMWR Morb Mortal Wkly Rep. 2002;51(38):853-6.

38. Abara WE, Hess KL, Neblett Fanfair R, Bernstein KT, Paz-Bailey G. Syphilis trends among men who have sex with men in the United States and Western Europe: a systematic review of trend studies published between 2004 and 2015. PLoS One. 2016;11(7):e0159309.

39. Baral SD, Poteat T, Stromdahl S, Wirtz AL, Guadamuz TE, Beyrer C. Worldwide burden of HIV in transgender women: a systematic review and meta-analysis. Lancet Infect Dis. 2013;13(3):214-22.

40. Bernstein KT, Chow JM, Pathela P, Gift TL. Bacterial sexually transmitted disease screening outside the clinic_-implications for the modern sexually transmitted disease program. Sex Transm Dis. 2016;43(2 Suppl 1):S42-52.

41. Oldenburg CE, Perez-Brumer AG, Reisner SL, Mattie J, Barnighausen T, Mayer KH, Mimiaga MJ. Global burden of HIV among men who engage in transactional sex: a systematic review and meta-analysis. PLoS One. 2014;9(7):e103549

42. Haddaway NR, Collins AM, Coughlin D, Kirk S. The role of Google Scholar in evidence reviews and its applicability to grey literature searching. PLoS One. 2015;10(9):e0138237.

43. Qi X, Yang M, Ren W, Jia J, Wang J, Han G, Fan D. Find duplicates among the PubMed, EMBASE, and Cochrane Library Databases in systematic review. PLoS One. 2013;8(8):e71838.

44. Rathbone J, Carter M, Hoffmann T, Glasziou P. Better duplicate detection for systematic reviewers: evaluation of Systematic Review Assistant-Deduplication Module. Syst Rev. 2015;4:6.

45. Kwon $Y$, Lemieux M, McTavish J, Wathen N. Identifying and removing duplicate records from systematic review searches. J Med Libr Assoc. 2015:103(4):184-8

46. Torgerson C. Systematic Reviews. London: Continuum; 2003.

47. Munn Z, Moola S, Lisy K, Riitano D, Tufanaru C. Methodological guidance for systematic reviews of observational epidemiological studies reporting prevalence and cumulative incidence data. Int J Evid Based Healthc. 2015;13(3):147-53.

48. Harder T. Some notes on critical appraisal of prevalence studies: Comment on: "The development of a critical appraisal tool for use in systematic reviews addressing questions of prevalence". Int J Health Policy Manag. 2014:3(5):289-90.

49. Egger M, Davey Smith G, Schneider M, Minder C. Bias in meta-analysis detected by a simple, graphical test. BMJ. 1997:315(7109):629-34

50. Guyatt GH, Oxman AD, Montori V, Vist G, Kunz R, Brozek J, Alonso-Coello P, Djulbegovic B, Atkins D, Falck-Ytter Y, et al. GRADE guidelines: 5. Rating the quality of evidence-publication bias. J Clin Epidemiol. 2011;64(12):1277-82.

\section{Submit your next manuscript to BioMed Central and we will help you at every step:}

- We accept pre-submission inquiries

- Our selector tool helps you to find the most relevant journal

- We provide round the clock customer support

- Convenient online submission

- Thorough peer review

- Inclusion in PubMed and all major indexing services

- Maximum visibility for your research

Submit your manuscript at www.biomedcentral.com/submit
Biomed Central 